\title{
Relay-Assisted Decorrelating Multiuser Detector (RAD-MUD) for Cooperative CDMA Networks
}

\author{
Wan-Jen Huang, Student Member, IEEE, Y.-W. Peter Hong, Member, IEEE, and C.-C. Jay Kuo, Fellow, IEEE,
}

\begin{abstract}
In this paper, we examine the uplink of a cooperative CDMA network, where users cooperate by relaying each other's messages to the base station. When spreading waveforms are not orthogonal, multiple access interference (MAI) exists at the relays and the destination, causing cooperative diversity gains to diminish. To address this issue, we adopt the multiuser detection (MUD) technique to mitigate MAI in achieving the full advantages of cooperation. Specifically, the relay-assisted decorrelating multiuser detector (RAD-MUD) is proposed to separate interfering signals at the destination with the help of precoding at the relays along with pre-whitening at the destination. Unlike the conventional zero-forcing (ZF) precoder or the decorrelating MUD, the proposed RAD-MUD experiences neither power expansion at the relays nor noise amplification at the destination. Three cooperative transmission strategies are considered on top of RAD-MUD; namely, transmit beamforming, selective relaying and distributed space-time coding. Since the reliability of each source-relay and/or relay-destination links are different, relay transmissions are weighted accordingly in our schemes to further combat MAI. The advantages of RAD-MUD over ZF precoding and other existing cooperative MUD schemes are shown through computer simulations.
\end{abstract}

Index Terms-Cooperative communications, multiuser detection, MIMO, precoding, space-time code.

\section{INTRODUCTION}

W IRELESS transmissions are subject to non-ideal channel effects such as multipath fading and multiple access interference (MAI). Many efforts have been made to mitigate these effects in order to improve the transmission efficiency. Specifically, cooperative communications [1]-[6] have been proposed to combat multipath fading by allowing users to relay each other's messages to the destination. Since each message may be transmitted through multiple relay paths, spatial diversity gains can be achieved without requiring multiple antennas on each communication terminal. In this work, we consider the uplink of a code-division-multipleaccess (CDMA) network in the context of cooperative communications, where, at a particular instant in time, a set of users serves as sources and another set of users serves as relays that forward the messages from the sources to the basestation. However, when the spreading waveforms adopted in

Manuscript received June 8, 2007; revised October 25, 2007. This work is supported in part by the National Science Council, Taiwan, under the grants NSC-96-2628-E-007-012-MY2, NSC-95-2221-E-007-043-MY3 and NSC-962219-E-007-009. This work was presented in part at GLOBECOM 2007, Washington, DC, USA.

Wan-Jen Huang and C.-C. Jay Kuo are with the Ming Hsieh Department of Electrical Engineering, University of Southern California, Los Angeles, CA 90089-2564, USA (e-mail: wanjenhu@usc.edu and cckuo@sipi.usc.edu).

Y.-W. Peter Hong is with the Institute of Communications Engineering, National Tsing Hua University, Hsinchu, Taiwan (e-mail: ywhong@ee.nthu.edu.tw).

Digital Object Identifier 10.1109/JSAC.2008.080413. the CDMA network are not orthogonal, MAI will exist at the relays and the destination, causing the cooperative diversity gains to diminish. To address this issue, multiuser detection (MUD) schemes must be adopted to mitigate MAI and to achieve the full advantages of cooperation.

Many cooperative communication strategies have been proposed in the literature based on relaying techniques, such as amplify-and-forward, decode-and-forward [3], coded cooperation [7], quantize-and-forward [8] etc. In this work, we focus on the decode-and-forward (DF) scheme where the relays decode and re-encode the sources' messages before retransmitting them to the destination. The relays form a distributed antenna array for each source using only one antenna at each node. Numerous communication and signal processing techniques originally developed for conventional multipleinput multiple-output (MIMO) systems, such as beamforming [9], [10], space-time coding [11], [12], antenna selection schemes [13], [14], can be applied to cooperative systems as well. These techniques will also be studied in this work.

Several multiuser detection (MUD) schemes [15] have been proposed to mitigate MAI in non-cooperative CDMA networks. Some well-known methods are, for example, the maximum likelihood (ML) detector, the decorrelating detector [16], the minimum-mean-square-error (MMSE) detector [17], the decision feedback detector [18] and the successive or parallel interference cancellation schemes [19]. The ML detector minimizes the error probability but has complexity that increases exponentially with the number of users. To address this issue, linear decorrelating and MMSE receivers, which require only polynomial complexity, have been proposed. However, the reduced computational complexity comes at the cost of higher bit-error-rates (BER). In particular, the decorrelating receiver eliminates MAI but may lead to noise amplification when spreading codes are non-orthogonal. The MMSE receiver controls the noise amplification up to a certain degree but results in higher residual MAI. Nonlinear decision-feedback and interference cancelation schemes offer good performance but experience large latency and error propagation.

Most existing works on cooperative communications assume that there is only one source in the network (while all other users serve as relays) or that there are multiple sources but each transmits over an orthogonal channel (which implies the availability of orthogonal spreading codes). However, in practical systems, the requirement for orthogonality is difficult to satisfy and, thus, the MAI cannot be ignored. More recently, MUD has been studied in [20], [21] for pair-wise cooperative systems, where each user is grouped with another user into a cooperative pair and is only allowed to forward messages 
transmitted by its dedicated partner. In other words, each relay helps forward the message from only one source and utilizes MUD to suppress signals transmitted by all other sources. The degree of freedom provided by the multiple relays and the relays' ability to jointly process the sources' messages are not fully exploited.

In this work, we consider a different scenario where each relay may cooperate with multiple users simultaneously. Messages received from multiple sources are decoded using the MMSE multiuser detector (MMSE-MUD) at the relays and are jointly processed before being retransmitted to the base station. By exploiting the relay's ability to preprocess messages, we propose the relay-assisted decorrelating multiuser detector (RAD-MUD) to separate (or to decorrelate) the multiple access interfering signals at the destination. Although the decorrelation of signals can also be achieved with either the zero-forcing $(\mathrm{ZF})$ precoder [22] or the decorrelating MUD [16], these schemes perform the decorrelating operations either entirely at the transmitter or entirely at the receiver, resulting in either power expansion at the transmitter or noise amplification at the receiver. Unlike $\mathrm{ZF}$ precoding or decorrelating MUD, RAD-MUD performs half of the decorrelating operation at the relays and half at the destination. This unique feature of RAD-MUD allows us to avoid both power expansion and noise amplification and, thus, results in better BER performances compared to existing cooperative MUD schemes. Three cooperative transmission schemes are considered on top of RADMUD; namely, transmit beamforming, selective relaying and distributed space-time coding (DSTC). Moreover, since the fading and MAI on each source-relay path (or each relaydestination path) is different, relay transmissions are weighted accordingly in our schemes to further combat MAI.

The rest of this paper is organized as follows. The model for a cooperative CDMA system is given in Section II and RADMUD is described in Section III. The MMSE MUD used at the relays and the signal combining methods at the destination are derived in Section IV. Three cooperative transmission strategies are examined in Section $\mathrm{V}$ and performance comparisons are shown in Section VI. Finally, concluding remarks are given in Section VII.

\section{SySTEM MOdEL}

Consider a cooperative network where $K$ users, denoted by $S_{1}, S_{2}, \cdots, S_{K}$, serve as sources and $L$ users, denoted by $R_{1}, R_{2}, \cdots, R_{L}$, serve as relays that forward messages from the sources to the destination as shown in Fig. 1. Each user is assigned a unique spreading code that is non-orthogonal but linearly independent from each other. The system performs two phases of transmission. In Phase I, the sources send their messages directly to the destination using their respective spreading codes. The transmissions are overheard and decoded by the relays in Phase II, and retransmitted to the destination using the same set of spreading codes. The transmissions from all users are assumed to be synchronous such that the transmitted symbols arrive at the receivers simultaneously. This simplifying assumption allows us to focus on the benefits of relaying in a multiuser system and the performance study provides bounds on the achievable performance in more practical settings.

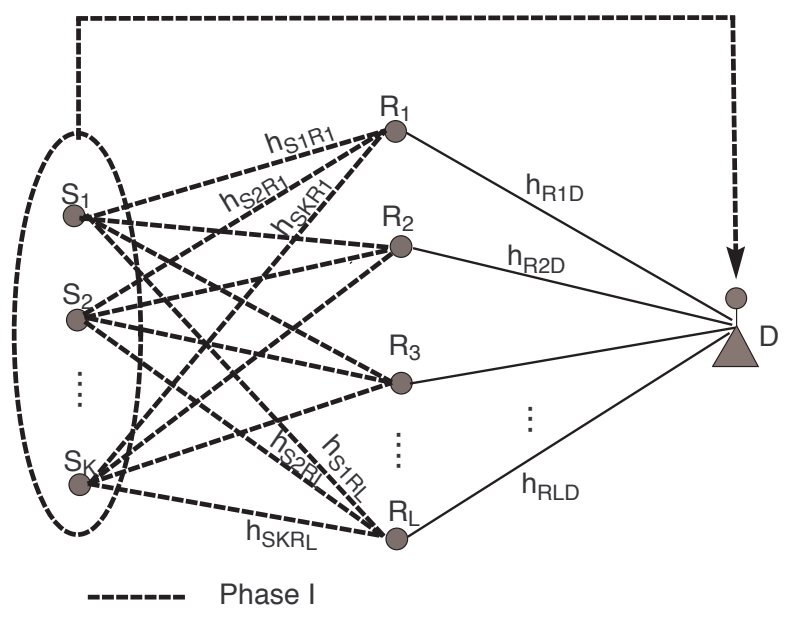

Phase II

Fig. 1. Cooperative CDMA uplink with $K$ sources and $L$ relays.

\section{Phase I - Direct Transmission Phase}

In Phase I, each source transmits a message with $M$ data symbols to the destination, denoted by $D$. Let $\mathbf{x}[m]=$ $\left[x_{1}[m], \cdots, x_{K}[m]\right]^{T}$ be the BPSK data symbols transmitted by sources $S_{1}, \cdots, S_{K}$ during the $m$-th symbol period, where $x_{k}[m] \in\{-1,1\}$ and

$$
\mathbf{E}\left[\mathbf{x}[m] \mathbf{x}[\ell]^{T}\right]= \begin{cases}\mathbf{I}_{K \times K}, & \text { if } m=\ell \\ \mathbf{0}_{K \times K}, & \text { otherwise }\end{cases}
$$

( $\mathbf{I}_{K \times K}$ denotes the $K \times K$ identity matrix and $\mathbf{0}_{K \times K}$ the $K \times K$ matrix with zeros in all elements.) Let $P_{S_{k}}$ be the power transmitted by source $S_{k}$ and let $s_{k}(t)$ be the spreading waveform of $S_{k}$. Under the synchronous assumption, the signal received at the destination during Phase $\mathrm{I}$ is

$$
y_{I}(t)=\sum_{m=1}^{M} \sum_{k=1}^{K} h_{S_{k} D} \sqrt{P_{S_{k}}} x_{k}[m] s_{k}\left(t-m T_{s}\right)+v_{I}(t),
$$

where $T_{s}$ is the symbol period, $h_{S_{k} D}$ is the complex channel coefficient from $S_{k}$ to $D$, and $v_{I}(t)$ is the additive white Gaussian noise (AWGN). We assume a block fading environment where channel coefficients remain constant over the $M$ symbol block and are independent and identically distributed (i.i.d.) from block to block. The channel coefficient $h_{S_{k} D}$ is assumed to be circularly symmetric complex Gaussian with 0 -mean and variance $\sigma_{S_{k} D}^{2}$, i.e., $h_{S_{k} D} \sim \mathcal{C N}\left(0, \sigma_{S_{k} D}^{2}\right)$, and is assumed to be independent among sources. If $N$ is the spreading gain, the spreading waveform for $S_{k}$ can be expressed as

$$
s_{k}(t)=\frac{1}{\sqrt{N}} \sum_{n=1}^{N} c_{k}[n] \varphi\left(t-n T_{c}\right), \quad k=1, \cdots, K,
$$

where $c_{k}[n]$ is the $n$-th element of the \pm 1 spreading sequence assigned to $S_{k}$, and $\varphi(t)$ is the normalized chip waveform with unit energy and duration $T_{c}=T_{s} / N$.

The received signal $y_{I}(t)$ at the destination $D$ is passed through a matched filter bank (MFB) corresponding to the spreading waveforms $s_{1}(t), \cdots, s_{K}(t)$, which are assumed known at both the relays and the destination. Let $\mathbf{R}$ be the 
correlation matrix of the spreading waveforms with the $(i, j)$ th element equal to

$$
[\mathbf{R}]_{i, j}=\int_{0}^{T_{s}} s_{i}(t) s_{j}(t) d t .
$$

The signals obtained at the output of the MFB during the $m$-th symbol period in Phase I is given by

$$
\mathbf{y}_{I}[m]=\mathbf{R H}_{S D} \mathbf{x}[m]+\mathbf{v}_{I}[m], \quad m=1, \cdots, M,
$$

where $\mathbf{H}_{S D}=\operatorname{diag}\left(\sqrt{P_{S_{1}}} h_{S_{1} D}, \sqrt{P_{S_{2}}} h_{S_{2} D}, \cdots, \sqrt{P_{S_{K}}} h_{S_{K} D}\right)$ and $\mathbf{v}_{I}[m]$ is the AWGN with distribution $\mathcal{C N}\left(\mathbf{0}_{K \times 1}, \sigma_{v}^{2} \mathbf{R}\right)$. The MFB outputs obtained over the entire symbol block is denoted by $\mathbf{Y}_{I}=\left[\mathbf{y}_{I}[1], \cdots, \mathbf{y}_{I}[M]\right]$.

\section{Phase II - Cooperative Transmission Phase}

Due to the broadcast nature of wireless channels, signals emitted by the sources in Phase I are also received at relays $R_{1}, R_{2}, \cdots, R_{L}$. Similar to (3), the signal received at $R_{l}$ is passed through an MFB and the output in the $m$-th symbol period is

$$
\mathbf{u}_{l}[m]=\mathbf{R H}_{S R_{l}} \mathbf{x}[m]+\mathbf{n}_{l}[m], \quad m=1, \cdots, M,
$$

where $\mathbf{H}_{S R_{l}}=\operatorname{diag}\left(\sqrt{P_{S_{1}}} h_{S_{1} R_{l}}, \cdots, \sqrt{P_{S_{K}}} h_{S_{K} R_{l}}\right)$ and $\mathbf{n}_{l}[m]$ is the AWGN at relay $R_{l}$ with covariance matrix $\sigma_{n}^{2} \mathbf{R}$. Here, $h_{S_{k} R_{l}} \sim \mathcal{C N}\left(0, \sigma_{S_{k} R_{l}}^{2}\right)$ is the channel coefficient between $S_{k}$ and $R_{l}$, and is assumed to be independent for each source-relay link. Based on the MFB output, data symbols are detected at each relay using the MMSE multiuser detector as to be described in Sec. IV. The block of symbols detected at $R_{l}$ are denoted by $\hat{\mathbf{X}}_{l}=\left[\hat{\mathbf{x}}_{l}[1], \cdots, \hat{\mathbf{x}}_{l}[M]\right]$, where $\hat{\mathbf{x}}_{l}[m]$ is the detection made at relay $R_{l}$ on the transmitted symbol vector $\mathbf{x}[m]$.

Depending on the cooperative strategy, the detected symbols $\hat{\mathbf{X}}_{l}$ may be re-encoded into the $K$-by- $M$ symbol matrix $\mathbf{T}_{l}=$ $f\left(\hat{\mathbf{X}}_{l}\right)=\left[\mathbf{t}_{l}[1], \cdots, \mathbf{t}_{l}[M]\right]$, where $\mathbf{t}_{l}[m]=\left[t_{l, 1}[m], \cdots, t_{l, K}[m]\right]^{T}$ is the symbol vector transmitted by $R_{l}$ during the $m$-th symbol period. When beamforming or selective relaying is considered [c.f. Sec. III], $\mathbf{t}_{l}[m]$ depends only on the detected symbols in the $m$-th time period, i.e., $\hat{\mathbf{x}}_{l}[m]$. When space-time codes are considered, $\mathbf{t}_{l}[m]$ will depend on the entire matrix $\hat{\mathbf{X}}_{l}$. During the $m$-th symbol period, $t_{l, k}[m]$ will be transmitted using the spreading waveform of source $S_{k}$ and the signal received at destination $D$ during Phase II is given by

$$
y_{I I}(t)=\sum_{m=1}^{M} \sum_{k=1}^{K} \sum_{l=1}^{L} h_{R_{l} D} t_{l, k}[m] s_{k}\left(t-m T_{s}\right)+v_{I I}(t),
$$

where $h_{R_{l} D}$ is the channel coefficient between $R_{l}$ and $D$ and $v_{I I}(t)$ is the AWGN. The power transmitted by $R_{l}$ in the $m$-th symbol period is given by

$$
\begin{aligned}
P_{R_{l}} & \triangleq \mathbf{E}\left[\int_{-\infty}^{\infty}\left|\sum_{k=1}^{K} t_{l, k}[m] s_{k}(t-m T)\right|^{2} d t\right] \\
& =\mathbf{E}\left[\mathbf{t}_{l}[m]^{H} \mathbf{R t}_{l}[m]\right],
\end{aligned}
$$

which is fixed over all symbol periods and must satisfy the total power constraint

$$
\sum_{l=1}^{L} P_{R_{l}} \leq P_{R}
$$

Similarly, in the $m$-th symbol period, the signal at $D$ is passed through the MFB to yield the output

$$
\mathbf{y}_{I I}[m]=\sum_{l=1}^{L} h_{R_{l} D} \mathbf{R t}_{l}[m]+\mathbf{v}_{I I}[m], \quad m=1, \cdots, M,
$$

where $h_{R_{l} D} \sim \mathcal{C N}\left(0, \sigma_{R_{l} D}^{2}\right)$ and $\mathbf{v}_{I I}[m] \sim \mathcal{C N}\left(\mathbf{0}_{K \times 1}, \sigma_{v}^{2} \mathbf{R}\right)$. The channel coefficients of each source-relay, relaydestination, and source-destination links are assumed to be independent among each other and i.i.d. over each block. The MFB output over the entire symbol block is $\mathbf{Y}_{I I}=\left[\mathbf{y}_{I I}[1], \cdots\right.$, $\left.\mathbf{y}_{I I}[M]\right]$. The signals received in Phase I and II are then combined at the destination to further increase diversity gains.

\section{Relay-Assisted Decorrelating Multiuser DETECTOR (RAD-MUD)}

As shown in (3) and (8), the signals obtained at the MFB output are subject to MAI if spreading waveforms are nonorthogonal. In this case, MUD can be employed at both the relays and the destination to mitigate MAI. For the non-cooperative CDMA system, the decorrelating MUD was proposed in [16] to eliminate MAI by multiplying the MFB output with the inverse of the correlation matrix. That is, for the signal received in Phase II, as given by (8), the decorrelator output at $D$ is equal to

$$
\mathbf{R}^{-1} \mathbf{y}_{I I}[m]=\sum_{l=1}^{L} h_{R_{l} D} \mathbf{t}_{l}[m]+\mathbf{R}^{-1} \mathbf{v}_{I I}[m] .
$$

In this case, the $k$-th term of the decorrelator output depends only on the symbols transmitted with the spreading code $s_{k}(t)$, i.e., the $k$-th term in each of the vectors $\mathbf{t}_{1}[m], \cdots, \mathbf{t}_{L}[m]$. Although this method eliminates MAI, the noise variance may increase due to the correlation among spreading codes. This is observed from the noise covariance matrix which is given by $\mathbf{E}\left[\mathbf{R}^{-1} \mathbf{v}_{I I}[m]\left(\mathbf{R}^{-1} \mathbf{v}_{I I}[m]\right)^{H}\right]=\sigma_{v}^{2} \mathbf{R}^{-1}$. To address this issue, we proposed the relay-assisted decorrelating multiuser detector (RAD-MUD) which, with the help of precoding at the relays, allows us to decorrelate the signals at the destination $D$ without noise enhancement.

The block diagram of RAD-MUD is illustrated in Fig. 2. Suppose that the relays (i.e., $R_{l}, l=1, \cdots, L$ ) have knowledge of the spreading codes of all sources. Each relay, say $R_{l}$, first encodes the detected symbol matrix $\hat{\mathbf{X}}_{l}$ into a $K$-by- $M$ matrix $g_{l}\left(\hat{\mathbf{X}}_{l}\right)$, where $g_{l}(\cdot)$ depends only on the specific cooperative transmission strategy employed, as discussed in Sec. V. For example, with beamforming, we have $g_{l}\left(\hat{\mathbf{X}}_{l}\right)=\mathbf{W}_{l} \hat{\mathbf{X}}_{l}$, where $\mathbf{W}_{l}$ is a diagonal matrix consisting of the beamforming coefficients. The output of the cooperative operation $g_{l}\left(\hat{\mathbf{X}}_{l}\right)$ is then precoded by the matrix $\mathbf{L}^{-H}$, where $\mathbf{L}$ is the Cholesky decomposition of $\mathbf{R}$ such that $\mathbf{R}=\mathbf{L} \mathbf{L}^{H}$ ( $\mathbf{L}$ is a $K$-by- $K$ lower triangular matrix). The symbol matrix transmitted by $R_{l}$ is given by

$$
\mathbf{T}_{l}=f\left(\hat{\mathbf{X}}_{l}\right)=\mathbf{L}^{-H} g_{l}\left(\hat{\mathbf{X}}_{l}\right) .
$$

The mapping $g_{l}(\cdot)$ must be chosen to satisfy the total power constraint in (6) and (7) such that

$$
\sum_{l=1}^{L} \mathbf{E}\left[\mathbf{t}_{l}[m]^{H} \mathbf{R} \mathbf{t}_{l}[m]\right]=\sum_{l=1}^{L} \mathbf{E}\left[g_{l}\left(\hat{\mathbf{X}}_{l}\right)[m]^{H} g_{l}\left(\hat{\mathbf{X}}_{l}\right)[m]\right] \leq P_{R},
$$




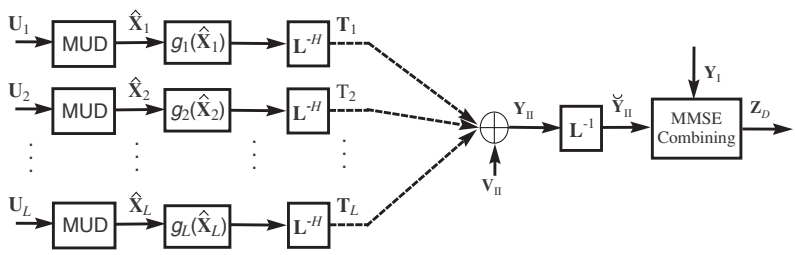

Fig. 2. The Block diagram of RAD-MUD

where $g_{l}\left(\hat{\mathbf{X}}_{l}\right)[m]$ is the $m$-th column of $g_{l}\left(\hat{\mathbf{X}}_{l}\right)$. It is worthwhile to note that, unlike the ZF precoding [22] scheme, the precoding employed in RAD-MUD as given in (9) does not result in power expansion since the transmitted power depends only on the cooperative transmission strategy and not on the correlation of the spreading codes.

With precoding at the relays, the MFB output at $D$ (which is obtained by substituting (9) into (8)) is given by

$$
\mathbf{Y}_{I I}=\sum_{l=1}^{L} h_{R_{l} D} \mathbf{R L}^{-H} g_{l}\left(\hat{\mathbf{X}}_{l}\right)+\mathbf{V}_{I I},
$$

where $\mathbf{V}_{I I}=\left[\mathbf{v}_{I I}[1], \cdots, \mathbf{v}_{I I}[M]\right]$. The received signal in (10) is then pre-multiplied with $\mathbf{L}^{-1}$, which yields the output

$$
\breve{\mathbf{Y}}_{I I}=\sum_{l=1}^{L} h_{R_{l} D} g_{l}\left(\hat{\mathbf{X}}_{l}\right)+\breve{\mathbf{V}}_{I I},
$$

where $\breve{\mathbf{Y}}_{I I}=\mathbf{L}^{-1} \mathbf{Y}_{I I}=\left[\breve{\mathbf{y}}_{I I}[1], \cdots, \breve{\mathbf{y}}_{I I}[M]\right]$ and $\breve{\mathbf{V}}_{I I}=\mathbf{L}^{-1} \mathbf{V}_{I I}$ $=\left[\breve{\mathbf{v}}_{I I}[1], \cdots \breve{\mathbf{v}}_{I I}[M]\right]$. It is worthwhile to point out that the noise term $\breve{\mathbf{v}}_{I I}[k]=\mathbf{L}^{-1} \mathbf{v}_{I I}[k]$ now has the covariance matrix $\mathbf{E}\left[\breve{\mathbf{v}}_{I I}[k] \breve{\mathbf{v}}_{I I}[k]^{H}\right]=\sigma_{v}^{2} \mathbf{I}_{K \times K}$ and, thus, $\mathbf{L}^{-1}$ can be viewed as a whitening filter. To conclude, with precoding at the relays and pre-whitening at $D$, the signals transmitted by different spreading codes are decorrelated at the destination without noise amplification or power expansion and, thus, constructing $K$ orthogonal channels between the relays and the destination.

\section{MMSE Multiuser Receivers ANd Signal COMBINING}

Although transmissions from relays to the destination can be decorrelated with RAD-MUD, signals received at the relays are still subject to MAI. In the following, we describe the use of MMSE-MUD at the relays ${ }^{1}$ and MMSE signal combining methods at $D$ to improve the system performance.

\section{A. MMSE Multiuser Detection at Relays}

As described in Sec. II, each relay receives an aggregate signal from the sources in Phase I and passes it through a MFB to obtain $\mathbf{u}_{l}[m]$, which is given in (4). At relay $R_{l}$, we apply the MMSE-MUD on $\mathbf{u}_{l}[\mathrm{~m}]$ and obtain the detected symbol vector

$$
\hat{\mathbf{x}}_{l}[m]=\operatorname{sgn}\left(\Re\left\{\mathbf{z}_{l}[m]\right\}\right)=\operatorname{sgn}\left(\Re\left\{\mathbf{C}_{l} \mathbf{u}_{l}[m]\right\}\right),
$$

where $\mathbf{z}_{l}[m]=\mathbf{C}_{l} \mathbf{u}_{l}[m]$ is the MMSE estimate of $\mathbf{x}[m]$ at relay $R_{l}$ and $\mathbf{C}_{l}$ is the $K \times K$ matrix chosen to minimize

\footnotetext{
${ }^{1}$ MMSE receivers are chosen here simply as a representative scheme. RADMUD is not reliant on any particular detection method at the relays.
}

the mean square error (MSE); namely, $\mathbf{E}\left[\left|\mathbf{C}_{l} \mathbf{u}_{l}[m]-\mathbf{x}[m]\right|^{2}\right]$. (Here, $\Re\{a\}$ denotes the real part of $a$; and, for $b$ that is real, $\operatorname{sgn}(b)=1$ if $b \geq 0$ and -1 if $b<0$.)

When the spreading codes are linearly independent and the correlation matrix $\mathbf{R}$ is of full rank, the MMSE solution can be computed as

$$
\begin{aligned}
\mathbf{C}_{l} & =\mathbf{E}\left[\mathbf{x}[m] \mathbf{u}_{l}[m]^{H}\right] \mathbf{E}\left[\mathbf{u}_{l}[m] \mathbf{u}_{l}[m]^{H}\right]^{-1} \\
& =\mathbf{H}_{S R_{l}}^{H} \mathbf{R}\left(\mathbf{R} \mathbf{H}_{S R_{l}} \mathbf{H}_{S R_{l}}^{H} \mathbf{R}+\sigma_{n}^{2} \mathbf{R}\right)^{-1},
\end{aligned}
$$

where index $m$ is omitted in $\mathbf{C}_{l}$ since symbols $\mathbf{x}[m]$ are assumed to be i.i.d. with respect to $m$ so that $\mathbf{C}_{l}$ is constant over time.

Let $\mathbf{C}_{l}=\left[\mathbf{c}_{1}^{(l)}, \mathbf{c}_{2}^{(l)}, \cdots, \mathbf{c}_{K}^{(l)}\right]^{T}$, where $\mathbf{c}_{k}^{(l)}=\left[c_{k, 1}^{(l)}, \cdots, c_{k, K}^{(l)}\right]^{T}$ for all $k$. The detected symbol corresponding to $S_{k}$ is $\hat{x}_{l, k}[m]=\operatorname{sgn}\left(\Re\left\{z_{l, k}[m]\right\}\right)=\operatorname{sgn}\left(\Re\left\{\left(\mathbf{c}_{k}^{(l)}\right)^{T} \mathbf{u}_{l}[m]\right\}\right)$. The BER of $S_{k}$ 's transmitted symbol at $R_{l}$ is given by

$$
\begin{aligned}
& \alpha_{l, k}=\frac{1}{2^{K-1}} \sum_{\substack{x_{i}[m] \in\{ \pm 1\} \forall i \neq k \\
x_{k}[m]=1}} \operatorname{Pr}\left(\Re\left\{z_{l, k}[m]\right\}<0 \mid x_{k}[m], x_{i}[m], \forall i \neq k\right)
\end{aligned}
$$

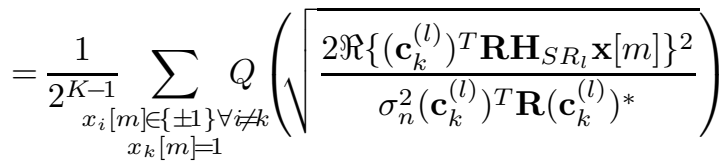

where $Q(x)=\int_{x}^{\infty} \frac{1}{\sqrt{2 \pi}} e^{-\frac{t^{2}}{2}} d t$.

When the number of interfering users is large, we can approximate the MAI contribution as Gaussian and the BER of the message transmitted by $S_{k}$ can be expressed as

$$
\begin{aligned}
\alpha_{l, k} & \cong Q\left(\sqrt{\frac{2\left[\left(\mathbf{c}_{k}^{(l)}\right)^{T} \mathbf{R H}_{S R_{l}}\right]_{k}^{2}}{\mathbf{E}\left[\left|z_{l, k}[m]\right|^{2}\right]-\left[\left(\mathbf{c}_{k}^{(l)}\right)^{T} \mathbf{R} \mathbf{H}_{S R_{l}}\right]_{k}^{2}}}\right) \\
& =Q\left(\sqrt{\frac{2\left[\boldsymbol{\Gamma}_{l}\right]_{k, k}}{1-\left[\boldsymbol{\Gamma}_{l}\right]_{k, k}}}\right),
\end{aligned}
$$

where

$$
\boldsymbol{\Gamma}_{l}=\mathbf{H}_{S R_{l}}^{H}\left(\mathbf{H}_{S R_{l}} \mathbf{H}_{S R_{l}}^{H}+\sigma_{n}^{2} \mathbf{R}^{-1}\right)^{-1} \mathbf{H}_{S R_{l}}
$$

and $[\mathbf{B}]_{i, j}$ is the $(i, j)$-th element of matrix $\mathbf{B}$.

\section{B. MMSE Signal Combining at Destination}

Based on the system model in Sec. II, $D$ receives two sets of signals: one directly from the sources in Phase I and the other from the relays in Phase II, as given in (3) and (8), respectively. When the precoding strategy at the relays and the channel coefficients on all links are known at $D$, signals from both the direct and the cooperative paths can be combined at $D$ to improve the detection performance. For example, sources that suffer from the near-far effect at certain relays may be detected reliably by others, therefore, diversity gains can be enhanced by properly weighting the signals received on each path.

Suppose that $\mathbf{T}_{l}=\mathbf{L}^{-H} g_{l}\left(\hat{\mathbf{X}}_{l}\right)$ is transmitted by $R_{l}$ and assume that $g_{l}\left(\hat{\mathbf{X}}_{l}\right)$ takes on the linear form $\mathbf{W}_{l} \hat{\mathbf{X}}_{l}(e . g$. in the case of beamforming and selective relaying [c.f. Sec. V]), where $\mathbf{W}_{l}=\operatorname{diag}\left(w_{l, 1}, \cdots, w_{l, K}\right)$ is a diagonal weighting matrix whose elements are determined by the transmission strategies and the total power constraint. To analyze errors in the 
detected symbol matrix, we define the random diagonal matrix $\boldsymbol{\Theta}_{l}[m]=\operatorname{diag}\left(\theta_{l, 1}[m], \cdots, \theta_{l, K}[m]\right)$ where $\theta_{l, k}[m] \in\{ \pm 1\}$ is a Bernoulli random variable with $\operatorname{Pr}\left(\theta_{l, k}[m]=-1\right)=\alpha_{l, k}$ and $\operatorname{Pr}\left(\theta_{l, k}[m]=1\right)=1-\alpha_{l, k}$. More specifically, we set $\theta_{l, k}[m]=1$ if the detection of $S_{k}$ 's $m$-th symbol at $R_{l}$ is correct and $\theta_{l, k}[m]=-1$ if it is incorrect $\left(\alpha_{l, k}\right.$ is equivalent to the BER). Therefore, the detected symbol matrix can be written as $\hat{\mathbf{X}}_{l}=\left[\hat{\mathbf{x}}_{l}[1], \cdots, \hat{\mathbf{x}}_{l}[M]\right]$, where

$$
\hat{\mathbf{x}}_{l}[m]=\boldsymbol{\Theta}_{l}[m] \mathbf{x}[m], \quad m=1,2, \ldots, M .
$$

The random variables $\theta_{l, k}[m]$ are $i . i . d$. over time with mean $\mathbf{E}\left[\theta_{l, k}[m]\right]=1-2 \alpha_{l, k}$. The signals received at $D$ in both phases can be expressed jointly as $\mathbf{Y}_{D}=\left[\begin{array}{ll}\mathbf{Y}_{I}^{T} & \breve{\mathbf{Y}}_{I I}^{T}\end{array}\right]^{T}=$ $\left[\mathbf{y}_{D}[1], \cdots, \mathbf{y}_{D}[M]\right]$, where

$$
\begin{aligned}
\mathbf{y}_{D}[m] & =\left[\begin{array}{c}
\mathbf{y}_{I}[m] \\
\breve{\mathbf{y}}_{I I}[m]
\end{array}\right] \\
& =\left[\begin{array}{c}
\mathbf{R H}_{S D} \\
\sum_{l=1}^{L} h_{R_{l} D} \mathbf{W}_{l} \boldsymbol{\Theta}_{l}[m]
\end{array}\right] \mathbf{x}[m]+\left[\begin{array}{c}
\mathbf{v}_{I}[m] \\
\breve{\mathbf{v}}_{I I}[m]
\end{array}\right]
\end{aligned}
$$

is the signal received in both phases during the $m$-th transmission period. Based on the signal given in (18), we can compute the MMSE multiuser detector as

$$
\hat{\mathbf{x}}_{D}[m]=\operatorname{sgn}\left(\Re\left\{\mathbf{z}_{D}[m]\right\}\right)=\operatorname{sgn}\left(\Re\left\{\mathbf{C}_{D} \mathbf{y}_{D}[m]\right\}\right),
$$

where $\mathbf{C}_{D}=\mathbf{E}\left[\mathbf{x}[m] \mathbf{y}_{D}^{H}[m]\right] \mathbf{E}\left[\mathbf{y}_{D}[m] \mathbf{y}_{D}^{H}[m]\right]^{-1}$.

Alternatively, to simplify the detection complexity, we first take the MMSE estimate of each source's symbol based on the signal received from the direct path, i.e., $\mathbf{y}_{I}[m]$. The MMSE estimate for each source obtained from the direct path is then combined with the corresponding decorrelated signal from the cooperative path using the MMSE criterion. Specifically, $D$ first computes the MMSE estimate of $\mathbf{x}[m]$ based on $\mathbf{y}_{I}[\mathrm{~m}]$ as

$$
\begin{aligned}
\mathbf{z}_{I}[m] & =\mathbf{E}\left[\mathbf{x}[m] \mathbf{y}_{I}[m]{ }^{H}\right] \mathbf{E}\left[\mathbf{y}_{I}[m] \mathbf{y}_{I}[m]^{H}\right]^{-1} \mathbf{y}_{I}[m] \\
& =\mathbf{H}_{S D}^{H} \mathbf{R}\left(\mathbf{R} \mathbf{H}_{S D} \mathbf{H}_{S D}^{H} \mathbf{R}+\sigma_{v}^{2} \mathbf{R}\right)^{-1} \mathbf{y}_{I}[m] .
\end{aligned}
$$

The $k$-th element of $\mathbf{z}_{I}[m]$ is the MMSE estimate of symbol $x_{k}[m]$, which is given by

$$
z_{I, k}[m]=\left[\boldsymbol{\Gamma}_{D}\right]_{k, k} x_{k}[m]+\xi_{k}[m],
$$

where $\boldsymbol{\Gamma}_{D}=\mathbf{H}_{S D}^{H}\left(\mathbf{H}_{S D} \mathbf{H}_{S D}^{H}+\sigma_{v}^{2} \mathbf{R}^{-1}\right)^{-1} \mathbf{H}_{S D}$ and $\xi_{k}[m]$ is the combined MAI-plus-Gaussian-noise term. By exploiting the fact that $x_{k}[m]$ is independent of $\xi_{k}[m]$ and $\mathbf{E}\left[\mathbf{z}_{I}[m] \mathbf{z}_{I}[m]^{H}\right]=\boldsymbol{\Gamma}_{D}$, one can show that $\xi_{k}[m]$ has zero mean and variance $\left[\boldsymbol{\Gamma}_{D}\right]_{k, k}-\left[\boldsymbol{\Gamma}_{D}\right]_{k, k}^{2}$.

The estimate of $S_{k}$ 's symbol received from both the direct and cooperative paths can be expressed as

$$
\begin{aligned}
\mathbf{y}_{D}^{(k)}[m] & \triangleq\left[\begin{array}{c}
z_{I, k}[m] \\
\breve{y}_{I I, k}[m]
\end{array}\right] \\
& =\left[\begin{array}{c}
{\left[\boldsymbol{\Gamma}_{D}\right]_{k, k}} \\
\sum_{l=1}^{L} h_{R_{l} D} w_{l, k} \theta_{l, k}[m]
\end{array}\right] x_{k}[m]+\left[\begin{array}{c}
\xi_{k}[m] \\
\breve{v}_{I I, k}[m]
\end{array}\right] \\
& =\mathbf{h}_{D}^{(k)} x_{k}[m]+\mathbf{v}_{D}^{(k)},
\end{aligned}
$$

where $\mathbf{h}_{D}^{(k)}=\left[\left[\boldsymbol{\Gamma}_{D}\right]_{k, k}, \quad \sum_{l=1}^{L} h_{R_{l} D} w_{l, k} \theta_{l, k}\right]^{T}$ and $\mathbf{v}_{D}^{(k)}=$ $\left[\xi_{k}[m], \breve{v}_{I I, k}[m]\right]^{T}$. The MMSE estimate of $x_{k}[m]$ is then computed based on the signal in (22), which is given by $z_{k}[m]=\mathbf{C}_{D}^{\prime} \mathbf{y}_{D}^{(k)}$ with

$$
\mathbf{C}_{D}^{\prime}=\mathbf{E}\left[x_{k}[m]\left(\mathbf{y}_{D}^{(k)}[m]\right)^{H}\right] \mathbf{E}\left[\mathbf{y}_{D}^{(k)}[m]\left(\mathbf{y}_{D}^{(k)}[m]\right)^{H}\right]^{-1}
$$

Note that

$\mathbf{E}\left[x_{k}[m]\left(\mathbf{y}_{D}^{(k)}[m]\right)^{H}\right]=\left[\left[\boldsymbol{\Gamma}_{D}\right]_{k, k}, \sum_{l=1}^{L} h_{R_{l} D}^{*} w_{l, k}^{*}\left(1-2 \alpha_{l, k}\right)\right]$ and

$$
\begin{aligned}
& \mathbf{E}\left[\mathbf{y}_{D}^{(k)}[m]\left(\mathbf{y}_{D}^{(k)}[m]\right)^{H}\right] \\
& \quad=\left[\begin{array}{cc}
\mathbf{E}\left[\left|z_{I, k}[m]\right|^{2}\right] & \mathbf{E}\left[z_{I, k}[m] \breve{y}_{I I, k}^{*}[m]\right] \\
\mathbf{E}\left[\breve{y}_{I I, k}[m] z_{I, k}^{*}[m]\right] & \mathbf{E}\left[\left|\breve{y}_{I I, k}[m]\right|^{2}\right]
\end{array}\right],
\end{aligned}
$$

where

$$
\begin{aligned}
& \mathbf{E}\left[\left|z_{I, k}[m]\right|^{2}\right]=\left[\boldsymbol{\Gamma}_{D}\right]_{k, k}, \\
& \mathbf{E}\left[z_{I, k}[m] \breve{y}_{I I, k}^{*}[m]\right]=\left[\boldsymbol{\Gamma}_{D}\right]_{k, k} \sum_{l=1}^{L} h_{R_{l} D}^{*} w_{l, k}^{*}\left(1-2 \alpha_{l, k}\right),
\end{aligned}
$$

and

$$
\begin{aligned}
& \mathbf{E}\left[\left|\breve{y}_{I I, k}[m]\right|^{2}\right] \\
& =\sum_{l=1}^{L} \sum_{l^{\prime}=1}^{L} h_{R_{l} D} h_{R_{l^{\prime}} D}^{*} w_{l, k} w_{l^{\prime}, k}^{*} \mathbf{E}\left[\theta_{l, k}[m] \theta_{l^{\prime}, k}[m]\right]+\sigma_{v}^{2} .
\end{aligned}
$$

The detection made on $S_{k}$ 's transmitted symbol is given by $\hat{x}_{D, k}[m]=\operatorname{sgn}\left(\Re\left\{z_{k}[m]\right\}\right)$. This method yields little performance loss compared with the previous method as shown through simulations in Sec. VI.

With RAD-MUD, it appears at first sight that the choice of spreading waveforms does not have a direct influence on symbol detection in Phase II. However, higher correlations between spreading waveforms do result in higher decoding BERs at the relays and, consequently, errors at the destination as well. The overall performance can be improved by choosing weighting factors that take into account detection reliability at relays as discussed in Sec. V.

Given the set of channel realizations

$$
\mathcal{H}=\left\{h_{S_{k} D}, h_{S_{k} R_{l}}, h_{R_{l} D} \text {, for } k=1, \cdots, K \text {, and } l=1, \cdots, L\right\}
$$

and correlation matrix $\mathbf{R}$, we can compute the BER of $S_{k}$ 's decoded symbols at $D$, which is denoted by $\mathrm{BER}_{k \mid \mathcal{H}, \mathbf{R}}$. As shown in (18), the detection performance depends on the specific error patterns at the relays, i.e., $\underline{\theta}_{k} \triangleq\left[\theta_{1, k}, \cdots, \theta_{L, k}\right]$. The conditional BER for decoding $S_{k}$ 's messages is

$$
\mathrm{BER}_{k \mid \mathcal{H}, \mathbf{R}}=\sum_{\underline{\theta}_{k} \in\{ \pm 1\}^{L}} \mathrm{BER}_{k \mid \mathcal{H}, \mathbf{R}, \underline{\theta}_{k}} \cdot \operatorname{Pr}\left(\underline{\theta}_{k} \mid \mathcal{H}, \mathbf{R}\right)
$$

where $\mathrm{BER}_{k \mid \mathcal{H}, \mathbf{R}, \underline{\theta}_{k}}$ is the BER conditioned on $\mathcal{H}, \mathbf{R}, \underline{\theta}_{k}$ and $\operatorname{Pr}\left(\underline{\theta}_{k} \mid \mathcal{H}, \mathbf{R}\right)$ is the probability of detection errors $\underline{\theta}_{k}$ given $\mathcal{H}$ and $\mathbf{R}$. By treating the interference as Gaussian, the conditional BER can be computed from (22) as

$$
\begin{aligned}
& \operatorname{BER}_{k \mid \mathcal{H}, \mathbf{R}, \underline{\theta}_{k}} \\
& =\operatorname{Pr}\left(\Re\left\{-\mathbf{C}_{D}^{\prime} \mathbf{h}_{D}^{(k)}+\mathbf{C}_{D}^{\prime} \mathbf{v}_{D}^{(k)}[m]\right\}>0 \mid x_{k}[m]=-1, \mathcal{H}, \mathbf{R}, \underline{\theta}_{k}\right) \\
& =Q\left(\sqrt{\operatorname{SINR}_{D, k}\left(\mathcal{H}, \mathbf{R}, \underline{\theta}_{k}\right)}\right) \cdot \mathbf{1}_{\left\{\Re\left\{\mathbf{C}_{D}^{\prime} \mathbf{h}_{D}^{(k)}\right\} \geq 0\right\}} \\
& +\left[1-Q\left(\sqrt{\operatorname{SINR}_{D, k}\left(\mathcal{H}, \mathbf{R}, \underline{\theta}_{k}\right)}\right)\right] \cdot \mathbf{1}_{\left\{\Re\left\{\mathbf{C}_{D}^{\prime} \mathbf{h}_{D}^{(k)}\right\}<0\right\}}
\end{aligned}
$$


where $\mathbf{1}_{\{B\}}$ represents the indicator function which is equal to 1 if the statement $B$ is true and 0 , otherwise, and the signalto-interference-plus-noise ratio is

$$
\operatorname{SINR}_{D, k}\left(\mathcal{H}, \mathbf{R}, \underline{\theta}_{k}\right)=\frac{\left(\Re\left\{\mathbf{C}_{D}^{\prime} \mathbf{h}_{D}^{(k)}\right\}\right)^{2}}{\frac{1}{2} \mathbf{E}\left[\mathbf{C}_{D}^{\prime} \mathbf{v}_{D}^{(k)}[m]\left(\mathbf{C}_{D}^{\prime} \mathbf{v}_{D}^{(k)}[m]\right)^{H}\right]},
$$

where

$$
\begin{aligned}
\Re\left\{\mathbf{C}_{D}^{\prime} \mathbf{h}_{D}^{(k)}\right\} & =\frac{[\boldsymbol{\Gamma}]_{k, k}}{1-[\boldsymbol{\Gamma}]_{k, k}}\left[\sigma_{v}^{2}+4 \sum_{l}\left|h_{R_{l} D} w_{l, k}\right|^{2}\left(\alpha_{l, k}-\alpha_{l, k}^{2}\right)\right] \\
& +\Re\left\{\sum_{l} \sum_{l^{\prime}} h_{R_{l} D} h_{R_{l^{\prime}}}^{*} w_{l, k} w_{l, k}^{*}\left(1-2 \alpha_{l^{\prime}, k}\right) \theta_{l, k}\right\},
\end{aligned}
$$

and

$$
\begin{aligned}
\mathbf{E}\left[\mathbf{C}_{D}^{\prime} \mathbf{v}_{D}^{(k)}[m]\left(\mathbf{C}_{D}^{\prime} \mathbf{v}_{D}^{(k)}[m]\right)^{H}\right] \\
=\frac{[\boldsymbol{\Gamma}]_{k, k}}{1-[\boldsymbol{\Gamma}]_{k, k}}\left[\sigma_{v}^{2}+4 \sum_{l}\left|h_{R_{l} D} w_{l, k}\right|^{2}\left(\alpha_{l, k}-\alpha_{l, k}^{2}\right)\right] \\
+\sigma_{v}^{2}\left|\sum_{l} h_{R_{l} D} w_{l, k}\left(1-2 \alpha_{l, k}\right)\right|^{2}
\end{aligned}
$$

The above derivation holds for all cooperative transmission strategies for which $g_{l}\left(\hat{\mathbf{X}}_{l}\right)=\mathbf{W}_{l} \hat{\mathbf{X}}_{l}$.

\section{Cooperative Transmission Strategies}

In RAD-MUD, the relay precoding allows us to construct $K$ orthogonal channels between the relays and the destination, similar to that in [2]. The message of each source is then transmitted through orthogonal channels with the help of a distributed antenna array formed by the relays. In this case, the cooperative signal processing techniques studied in the literature can be readily applied to this system. Based on the availability of channel state information (CSI), we discuss and compare the performance of three cooperative transmission strategies: (a) transmit beamforming; (b) selective relaying; and (c) distributed space-time coding. For transmit beamforming and selective relaying schemes, symbols transmitted by $R_{l}$ can be expressed in linear form as $g_{l}\left(\hat{\mathbf{X}}_{l}\right)=\mathbf{W}_{l} \hat{\mathbf{X}}_{l}$. To maintain fairness, we shall assume that the same total relay power is used to retransmit the detected symbols corresponding to each source, i.e., $\sum_{l=1}^{L}\left|w_{l, k}\right|^{2}=P_{R} / K$, for all $k$.

\section{A. Transmit Beamforming}

When full CSI is available to all relays, transmit beamforming can be applied, treating the relays as multiple antennas, to compensate for the phase differences on each relay path and obtain a coherent addition of signals at $D$. Here, we consider two beamforming methods.

1) Beamforming for Relay-Destination (R-D) Channels: In this scheme, beamforming coefficients at each relay are chosen based only on the values of the relay-destination ( $R$ D) channels. This is the solution that maximizes the signalto-noise ratio at the destination when the antennas are colocated at a single terminal, or, when the errors at the relays are negligible. For transmit beamforming schemes, we write $g_{l}\left(\hat{\mathbf{X}}_{l}\right)=\mathbf{W}_{l} \hat{\mathbf{X}}_{l}$ where $\mathbf{W}_{l}=\operatorname{diag}\left(w_{l, 1}, \cdots, w_{l, K}\right)$ contains the beamforming coefficients for each source at $R_{l}$ on the diagonal. Specifically, we have

$$
w_{l, k}=\beta_{\mathrm{RD}} h_{R_{l} D}^{*}, \quad \forall l, k,
$$

where $\beta_{\mathrm{RD}}=\sqrt{P_{R} /\left(K \sum_{l=1}^{L}\left|h_{R_{l} D}\right|^{2}\right)}$ is chosen to meet the power constraint $\sum_{l=1}^{L}\left|w_{l, k}\right|^{2}=P_{R} / K$.

2) Beamforming for Source-Relay-Destination ( $S-R-D)$ Channels: If errors at the relays are not negligible, one must consider the detection reliability at each relay when deriving the beamforming coefficients. Let $\mathbf{w}_{k}=\left[w_{1, k}, \cdots, w_{L, k}\right]^{T}$ be the beamforming coefficients used for retransmitting $S_{k}$ 's detected symbols at relays $R_{1}, \cdots, R_{L}$. To take into consideration the reliability of the relay detection, we choose $\mathbf{w}_{k}$ to minimize the MMSE at the destination for the signals corresponding to $S_{k}$ in Phase II. Specifically, with the derivations given in Appendix A, we obtain the optimal coefficients as

$$
\begin{aligned}
\mathbf{w}_{k} & =\arg \min _{\mathbf{w}_{k}}\left\{\min _{c_{k}} \mathbf{E}\left[\left|c_{k} \breve{y}_{I I, k}[m]-x_{k}[m]\right|^{2}\right]\right\} \\
& =\beta_{k, S R D}\left(\mathbf{\Phi}_{k}+\frac{K \sigma_{v}^{2}}{P_{R}} \mathbf{I}_{L \times L}\right)^{-1} \mathbf{p}_{k} .
\end{aligned}
$$

where $\mathbf{p}_{k}=\left[h_{R_{1} D}\left(1-2 \alpha_{1, k}\right), \cdots, h_{R_{L} D}\left(1-2 \alpha_{L, k}\right)\right]^{H}$ and $\boldsymbol{\Phi}_{k}$ is an $L \times L$ matrix with elements

$$
\left[\boldsymbol{\Phi}_{k}\right]_{l, l^{\prime}}=\left\{\begin{array}{cc}
\left|h_{R_{l} D}\right|^{2}, & \text { if } l=l^{\prime} \\
h_{R_{l} D}^{*} h_{R_{l^{\prime}} D} \mathbf{E}\left[\theta_{l, k} \theta_{l^{\prime}, k}\right], & \text { if } l \neq l^{\prime}
\end{array}\right.
$$

Here, $\beta_{k, S R D}=\sqrt{\frac{P_{R} / K}{\mathbf{p}_{k}^{H}\left(\mathbf{\Phi}_{k}+\frac{K \sigma_{v}^{2}}{P_{R}} \mathbf{I}_{L \times L}\right)^{-2} \mathbf{p}_{k}}}$ is chosen to meet the power constraint $\sum_{l=1}^{L}\left|w_{l, k}\right|^{2}=P_{R} / K$. Notice that, in $\mathbf{p}_{k}$, smaller weights are given to relays with larger decoding errors and/or less reliable R-D channels.

\section{B. Selective Relaying}

When only partial CSI is available at the relays, we consider two selective relaying strategies as detailed below.

1) Threshold Selection Strategy: Assume that each relay has the knowledge of only the local S-R and R-D channel coefficients. In this strategy, the relays decide independently whether it will relay a particular source's message using a locally selected threshold. Suppose that $\operatorname{SINR}_{S_{k} R_{l}}$ is the signalto-MAI-plus-noise ratio for $S_{k}$ 's transmitted signal measured at $R_{l}$ and $\mathrm{SNR}_{R_{l} D}$ is the signal-to-noise ratio at the destination for the signal transmitted by $R_{l}$. Then, for

$$
\begin{aligned}
\gamma_{l, k} & =\min \left\{\operatorname{SINR}_{S_{k} R_{l}}, \mathrm{SNR}_{R_{l}, D}\right\} \\
& =\min \left\{\frac{\left[\boldsymbol{\Gamma}_{l}\right]_{k, k}}{1-\left[\boldsymbol{\Gamma}_{l}\right]_{k, k}}, \frac{\beta_{T h, k}^{2}\left|h_{R_{l} D}\right|^{2}}{\sigma_{v}^{2}}\right\},
\end{aligned}
$$

where $\operatorname{SINR}_{S_{k} R_{l}}$ and $\boldsymbol{\Gamma}_{l}$ are given in (15), $R_{l}$ relays the message transmitted by $S_{k}$ if and only if $\gamma_{l, k}$ is greater or equal to the threshold $\gamma_{T}$. That is, $w_{l, k}=\beta_{T h, k}$ if $\gamma_{l, k} \geq \gamma_{T}$ and 0 , otherwise. The reasoning behind this selection criterion is that, since the BER of $S_{k}$ 's symbol at the destination is dominated by the maximum error probability between the $\mathrm{S}$ $\mathrm{R}$ link and the R-D link, we allow $R_{l}$ to relay the symbol only if the maximum error probability of the two links are 
sufficiently reliable, i.e., when the minimum SNR or SINR exceeds a certain threshold.

Note that, since only the local channel coefficients are known at each relay, $\beta_{T h, k}$ can only be chosen to satisfy the average power constraint $\sum_{l=1}^{L} \mathbf{E}\left[\left|w_{l, k}\right|^{2}\right]=P_{R} / K$. The threshold $\gamma_{T}$ is chosen numerically to minimize the average BER at the destination in Sec. VI. Furthermore, the threshold selection strategy can be combined with the beamforming RD strategy. In this case, only the SINR on the S-R link is used for comparison since full CSI of the R-D channel is available. Specifically, we set $w_{l, k}=\beta_{T h-B F} h_{R_{l} D}^{*}$ if $\mathrm{SINR}_{S_{k} R_{l}} \geq \gamma_{T}$, and $w_{l, k}=0$, otherwise. This method can be viewed as a compromise between Beamforming R-D and Beamforming S$\mathrm{R}-\mathrm{D}$ in terms of complexity and performance.

2) Best Selection Strategy: When global CSI is available but phase coherent transmission is not achievable due to imperfect synchronization, we can employ the best selection strategy where only one relay is chosen to transmit for each source. In this case, we set $w_{k, l}=\beta_{T h, k}$ if $\gamma_{l, k} \geq \gamma_{l^{\prime}, k}, \forall l^{\prime}$, and $w_{i, k}=0$, otherwise, where $\beta_{T h, k}=\sqrt{P_{R} / K}$. When the relays have only the local CSI, the selection can be conducted at the destination in a centralized manner or, distributedly, by using the opportunistic carrier sensing method proposed in [14]. The best selection strategy has been studied extensively in the literature and has been shown to achieve full diversity in the absence of MAI. In Appendix B, we show that full diversity can also be achieved with RAD-MUD even in the presence of MAI.

\section{Distributed Space-Time Coding (DSTC)}

When no CSI is available at the relays, we can employ distributed space-time coding (DSTC) [2], [11], [12] to exploit diversity in the cooperative system.

In particular, we adopt the linear dispersive space time code [23], where the space time code is obtained by multiplying the symbol block with a random unitary matrix generated independently at each relay. Suppose that DSTC is applied to $M$ consecutive symbols at each relay, where $M$ is chosen to be greater than $L$ in order to achieve full diversity [23]. Let $\mathbf{A}_{l}$ be the unitary and isotropic random matrix of dimension $M \times M$ used to encode the detected symbols from each source. The transmitted symbol matrix is then given by

$$
\mathbf{T}_{l}=\mathbf{L}^{-H} \mathbf{W}_{l} \hat{\mathbf{X}}_{l} \mathbf{A}_{l}, \quad l=1, \cdots, L .
$$

Since no CSI is available at relays, all diagonal elements of the weighting matrix is set to the same value. At the destination, the MMSE estimate obtained on the direct path and the signal received from the orthogonal cooperative path for each source is combined together to perform the maximum likelihood (ML) detection. (The residual MAI of the MMSE estimate is treated as Gaussian when applying the ML in Sec. VI.) Note that the MMSE detector is not used here since it does not achieve diversity in the case that we consider [c.f. [24]].

Furthermore, if only local CSI of the S-R links is available at relays, we can also combine the threshold selection strategy with DSTC so that $w_{l, k}=\beta_{D S T C}$ if and only if $\operatorname{SINR}_{S_{k} R_{l}}>$ $\gamma_{T}$. Similarly, $\beta_{D S T C}$ is chosen to satisfy the average power constraint as in Sec. V-B.

\section{PERformance COMPARISONS AND NuMERICAL SiMUlations}

The performance of RAD-MUD with various cooperation methods discussed in Sec.V is studied by computer simulation in this section. In these experiments, the channel coefficients $h_{S_{k} R_{l}}$ are assumed to be i.i.d. for all $k$ and $l$ with distribution $\mathcal{C N}(0,1)$. We assume that all sources and relays have equal distance to $D$ such that $h_{S_{k} D}, h_{R_{l} D} \sim \mathcal{C N}(0,1 / 16), \forall k$ and $l$. This corresponds to the case where sources and relays are located in the vicinity of each other and are all sufficiently far from the destination such that the distances to the destination can be considered as equal. The variance of the AWGN is equal to 1 for all receivers. Each source transmits with equal power $P_{s}$ and the sum transmit power of the sources is equal to the sum transmit power of the relays, i.e., $K P_{s}=P_{R}=P$. The spreading waveforms are generated randomly with nonsingular correlation matrices. The spreading gain is $N=8$.

For a cooperative network with $K=8$ sources and $L=8$ relays, we first compare, in Fig. 3, the proposed RAD-MUD with three different transmission schemes: (i) Zero-Forcing Precoding [22]; (ii) Cao \& Vojcic Cooperative MUD [20]; and (iii) Cooperative MMSE-MUD without precoding at the relays (Cooperative MMSE-MUD). In all of these schemes, MMSE-MUD is used at the relays to decode the messages from all sources. At the destination, MMSE multiuser detector is derived using the assumption that $\theta_{l, k}$ is independent over relays for simplicity, which is suboptimal detection. In scheme (i), each relay performs $\mathrm{ZF}$ precoding (as proposed in [22]) before the messages are retransmitted to the destination. That is, the symbol transmitted by $R_{l}$ is $\mathbf{T}_{l}=\beta_{z f} \mathbf{R}^{-1} g_{l}\left(\hat{\mathbf{X}}_{l}\right)$ where $\beta_{z f}$ is chosen to satisfy the total power constraint in (7). In scheme (ii), each relay forwards only the message of one source (i.e., its dedicated partner) while, in scheme (iii), each relay decodes-and-forwards the messages from all sources, similar to RAD-MUD, but do not perform precoding at the relays, i.e., $\mathbf{T}_{l}=g_{l}\left(\hat{\mathbf{X}}_{l}\right)$. The cooperative source-relay pairs for scheme (ii) are chosen randomly in our experiments. To focus on the advantages of relay-assisted decorrelation, the BER performances in Fig. 3 are shown without combining the signal on the direct path since the signal is common for all schemes. Moreover, we assume that all relays transmit with equal power and the weighting factors of all relayed symbols are identical for scheme (iii) and the RAD-MUD, i.e., $g_{l}\left(\hat{\mathbf{X}}_{l}\right)=\mathbf{W}_{l} \hat{\mathbf{X}}_{l}$, where $\mathbf{W}_{l}=\sqrt{P / K L} \mathbf{I}_{K \times K}, \forall l$. It is worthwhile to notice that the system with $\mathrm{ZF}$ Precoding at the relays achieves the same performance as the system that employs Decorrelating MUD at the destination without precoding at the relays; therefore, the latter is not shown redundantly in the figure. Although the signals received at the destination are decoupled for both ZF Precoding and RADMUD, we can see that RAD-MUD outperforms ZF Precoding by $7 \mathrm{~dB}$ since no power expansion occurs at the relays. We also show that RAD-MUD outperforms both scheme (ii) and scheme (iii) since the signals received at the destination are free of MAI.

In Figs. 4, 5 and 6, we show the performance of RAD-MUD with the different cooperative transmission strategies described in Section V. Since the Cooperative MMSE MUD of scheme 


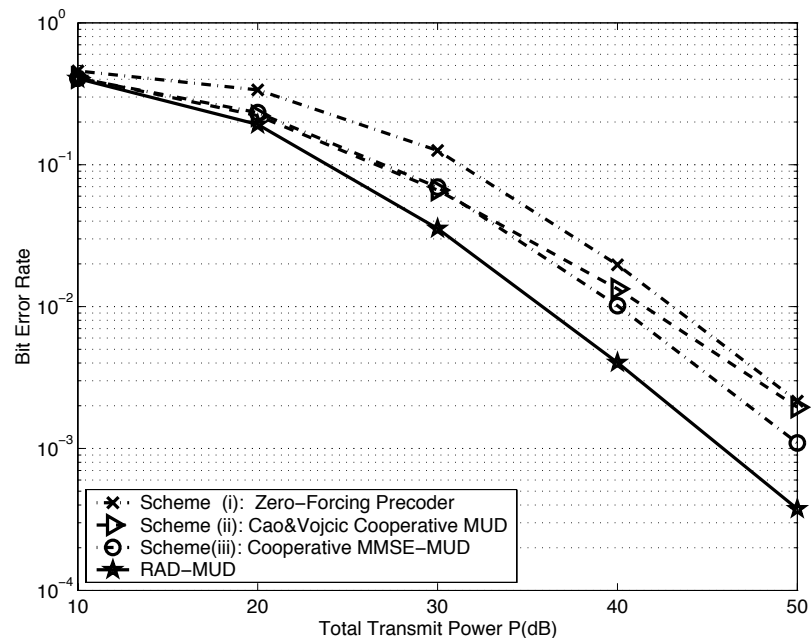

Fig. 3. BER performance comparison for different precoding and MUD strategies at the relays

(iii) outperforms schemes (i) and (ii), as shown in Fig. 3, and has a structure that is able to incorporate the cooperative transmission strategies (i.e., each relay in scheme (iii) is allowed to decode-and-forward messages from all sources), we shall compare RAD-MUD only with the Cooperative MMSE-MUD in the following experiments. The performance of the direct transmission with total transmit power set to $2 P$ is plotted as a reference. Here, we also consider a cooperative network with $K=8$ number of sources and $L=8$ number of relays. The signals received at the destination in both phases are combined using the low complexity MMSE combining method given in (23). The BER performance versus the total transmit power $P$ are shown in Figs. 4, 5 and 6 for transmit beamforming, selective relaying and DSTC, respectively. These methods are utilized to achieve the cooperative diversity gains in addition to reducing MAI with MUD. We can see that the RADMUD outperforms the Cooperative MMSE-MUD in all cases as detailed below. The improvement under RAD-MUD is most pronounced for cooperative transmission strategies that yield large cooperative diversity gains since, in these cases, MAI may dominate the BER and thus, it becomes increasingly important to effectively mitigate MAI. For conciseness, the word beamforming is abbreviated as "BF" and selective as "SEL" in the legend.

In Fig. 4, we compare the performance of Cooperative MMSE-MUD and RAD-MUD for three beamforming schemes: Beamforming S-R, Beamforming S-R-D and Beamforming with threshold selection. We can see that, by weighting the relay symbols by the reliability of the detection at the relays in Beamforming S-R-D, we can significantly improve the BER performance over Beamforming R-D. The Beamforming with threshold selection serves as a compromise between the above two schemes since it allows a relay to forward the symbol only when the detection is reliable. The improvement due to RAD-MUD is largest for beamforming S-R-D since this scheme yields the largest cooperative gain. In Fig. 5, the threshold selection and the best selection strategies are compared, where $\gamma_{T}$, in the threshold selection strategy, is obtained numerically to minimize the average of BER at $D$.

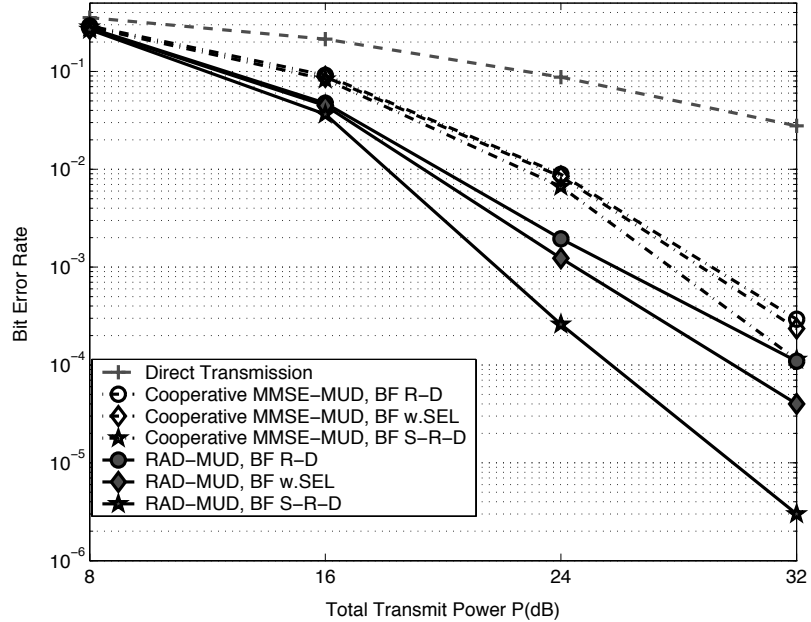

Fig. 4. BER performance comparison for systems employing transmit beamforming.

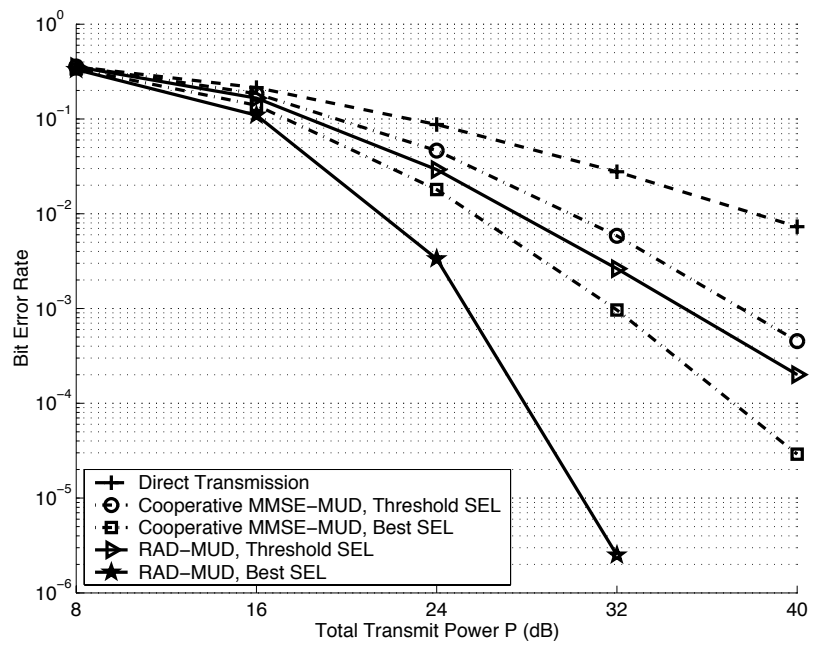

Fig. 5. BER performance comparison for systems employing selective relaying.

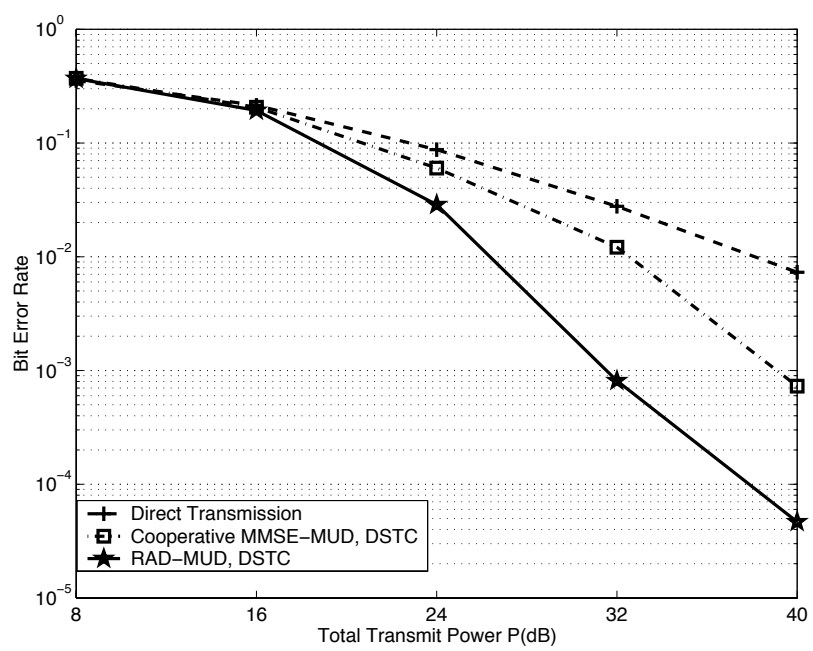

Fig. 6. BER performance comparison for systems employing DSTC. 


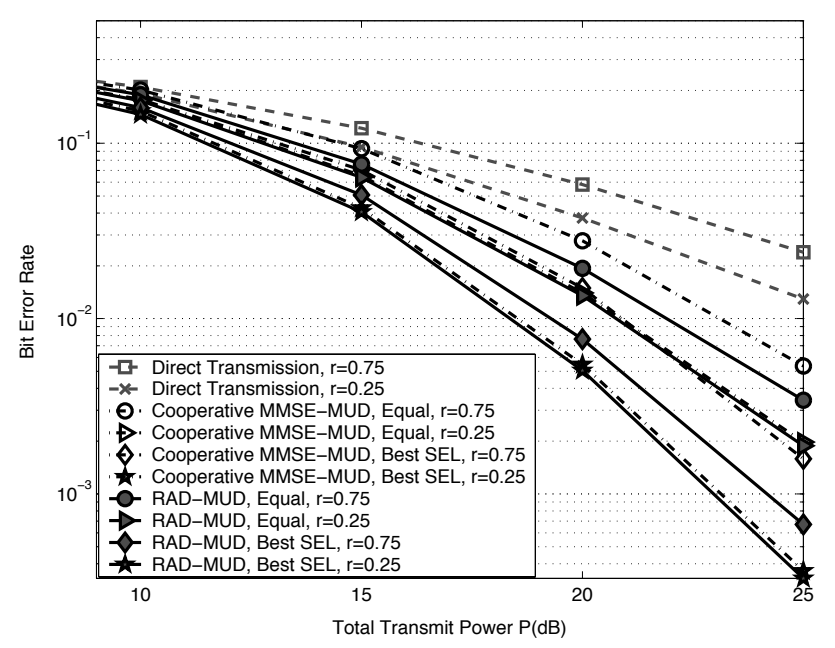

Fig. 7. BER comparison of the case with two users and two relays with correlation coefficients $\rho=0.75$ and 0.25 .

We can see that best selection outperforms threshold selection since it utilizes global CSI. More interestingly, the increase in diversity order for the best selection scheme is larger for RADMUD than Cooperative MMSE-MUD since the performance is less restricted by MAI. When DSTC is applied, as shown in Fig. 6, the RAD-MUD outperforms the Cooperative MMSEMUD by $7 \mathrm{~dB}$ when $\mathrm{BER}=10^{-3}$.

In Fig. 7, we consider the case with $K=L=2$ and compare the BER performance for $\rho=0.25$ and 0.75 , where $\rho=\int s_{1}(t) s_{2}(t) d t$ is the correlation between the two spreading codes. The direct transmission, equal gain and best selection methods are given for both Cooperative MMSE-MUD and RAD-MUD. As shown in the figure, RAD-MUD effectively combats the MAI when the correlation between two spreading codes is high, i.e., $\rho=0.75$. When $\rho=0.25$, the MAI is small and both RAD-MUD and Cooperative MMSE-MUD have comparable performance.

In Fig. 8, the BER of the best selection scheme is shown for $K=8$ and $L=2,4,8$. We can see that the increase in diversity is more evident for RAD-MUD as the number of relays increases. When $\mathrm{BER}=10^{-4}, 3.5 \mathrm{~dB}$ improvement is observed as $L$ increases from 2 to 8 for Cooperative MMSEMUD, while $7 \mathrm{~dB}$ is observed for RAD-MUD. To show the effectiveness of the low complexity MMSE combining method of (23), we compare the BER performance of the optimal MMSE combining (dashed-line) and the alternative lowcomplexity method (solid-line) in Fig. 9 for the equal gain, selective relaying, and beamforming strategies. As shown in the figure, the alternative method yields only little performance loss while requiring a much lower decoding complexity.

\section{CONCLUSION}

The relay-assisted decorrelating multiuser detector (RADMUD) was proposed to decouple users' signals at the basestation without noise amplification. This is achieved by precoding transmitted messages at the relays along with prewhitening of the received signals at the destination. Three cooperative transmission schemes were studied on top of the RAD-MUD system. They are transmit beamforming, selective

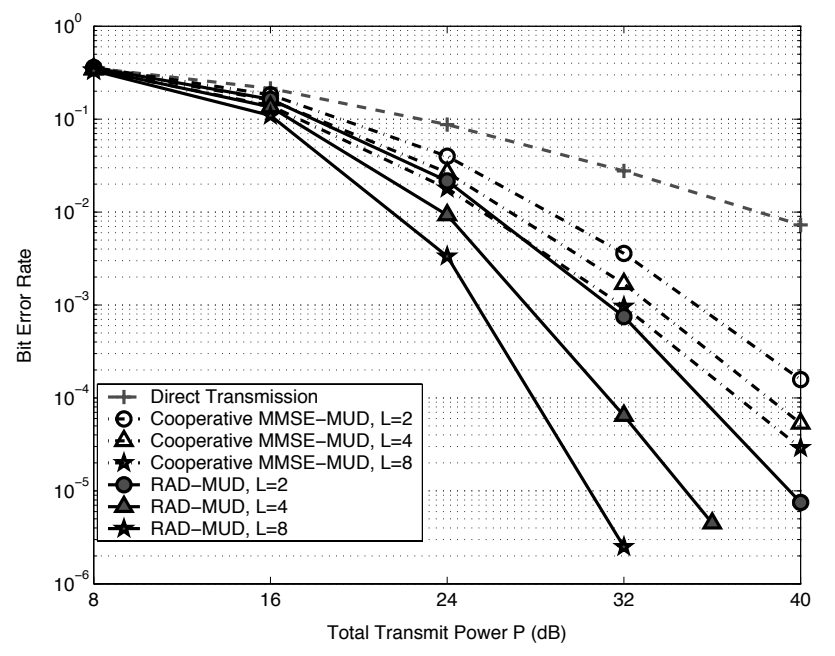

Fig. 8. BER versus total transmit power for $K=8$ and $L=2$ (circles), 4 (triangles) and 8 (stars).

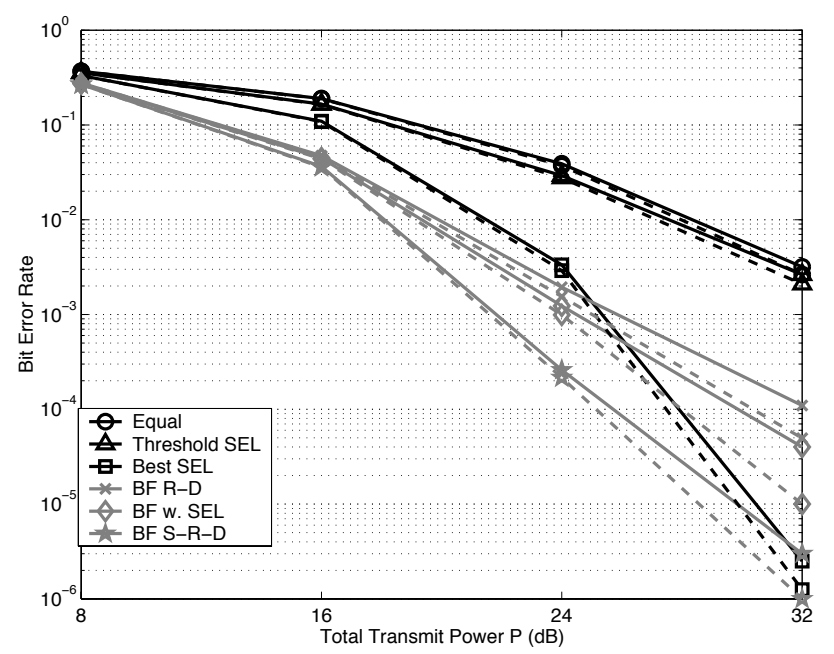

Fig. 9. Performance comparison between MMSE joint decoding in (19) (dashed line) and the alternative low-complexity MMSE combining in (23) (solid line).

relaying, and distributed space time coding. The performance of the proposed RAD-MUD scheme was demonstrated by computer simulation in representative test cases and is shown to outperform cases where cooperative MUD is used without relay-assisted decorrelation.

\section{APPENDIX A}

\section{DERIVATION OF BEAMFORMING COEFFICIENTS IN (25)}

Here, we derive the beamforming coefficients given in (25). Given $\breve{y}_{I I, k}[m]$, the MMSE estimate of the symbol $x_{k}[m]$ is computed and yields the MSE as

$$
\begin{aligned}
& \min _{c_{k}} \mathbf{E}\left[\left|c_{k} \breve{y}_{I I, k}[m]-x_{k}[m]\right|^{2}\right] \\
& =\mathbf{E}\left|x_{k}[m]\right|^{2}-\frac{\left|\mathbf{E}\left[x_{k}[m] \breve{y}_{I I, k}^{*}[m]\right]\right|^{2}}{\mathbf{E}\left|\breve{y}_{I I, k}^{*}[m]\right|^{2}}=1-\frac{\left|\mathbf{p}_{k}^{H} \mathbf{w}_{k}\right|^{2}}{\mathbf{w}_{k}^{H} \mathbf{\Phi}_{k} \mathbf{w}_{k}+\sigma_{v}^{2}},
\end{aligned}
$$

where $\mathbf{p}_{k}$ and $\boldsymbol{\Phi}_{k}$ are defined as in Sec. V-A. Under the power constraint that $\left|\mathbf{w}_{k}\right|^{2}=P_{R} / K, \forall k$, the beamforming 
coefficients are given by

$$
\mathbf{w}_{k}=\underset{\mathbf{w}:|\mathbf{w}|^{2}=\frac{P_{R}}{K}}{\arg \min _{\mathbf{w}^{H}\left(\boldsymbol{\Phi}_{k}+\frac{K \sigma_{v}^{2}}{P_{R}} \mathbf{I}\right) \mathbf{w}} .}
$$

Then, by defining $\mathbf{u}=\left(\boldsymbol{\Phi}_{k}+\frac{K \sigma_{v}^{2}}{P_{R}} \mathbf{I}\right)^{\frac{1}{2}} \mathbf{w}$, the optimization problem can be expressed as

$$
\mathbf{u}_{k}=\underset{\mathbf{u}}{\operatorname{argmin}} 1-\left|\mathbf{p}_{k}^{H}\left(\boldsymbol{\Phi}_{k}+\frac{K \sigma_{v}^{2}}{P_{R}} \mathbf{I}\right)^{\frac{-1}{2}} \frac{\mathbf{u}}{|\mathbf{u}|}\right|^{2} .
$$

By Cauchy-Schwarz inequality, the minimum of the MSE is attained when $\mathbf{u}_{k}$ is given by

$$
\mathbf{u}_{k}=\beta_{k, S R D}\left(\boldsymbol{\Phi}_{k}+\frac{K \sigma_{v}^{2}}{P_{R}} \mathbf{I}\right)^{\frac{-1}{2}} \mathbf{p}_{k},
$$

where $\beta_{k, S R D}$ is a non-zero arbitrary constant. It follows that

$$
\mathbf{w}_{k}=\beta_{k, S R D}\left(\boldsymbol{\Phi}_{k}+\frac{K \sigma_{v}^{2}}{P_{R}} \mathbf{I}_{L \times L}\right)^{-1} \mathbf{p}_{k}
$$

and, to satisfy the power constraint, we set

$$
\beta_{k, S R D}=\sqrt{\frac{P_{R} / K}{\mathbf{p}_{k}^{H}\left(\boldsymbol{\Phi}_{k}+\frac{K \sigma_{v}^{2}}{P_{R}} \mathbf{I}_{L \times L}\right)^{-1} \mathbf{p}_{k}}}
$$

\section{APPENDIX B \\ Diversity of The Best Selection Strategy}

The best selection strategy in RAD-MUD achieves full diversity as shown in the following. Without loss of generality, let source $S_{1}$ be the user of interest, and $R_{l^{*}}$ be the selected relay. For simplicity, we choose $P_{S}=P_{R} / K=P$ and $\sigma_{S_{k} R_{l}}^{2}=\sigma_{R_{l} D}^{2}=\sigma_{v}^{2}=\sigma_{n}^{2}=1, \forall k$ and $l$. Meanwhile, we consider the case where $[\mathbf{R}]_{i, j}=\rho<1$, for $i \neq j$ and $[\mathbf{R}]_{i, i}=1$, for all $i$ (i.e., the set of spreading codes is generated using the shifted versions of $\mathrm{m}$-sequences [25]). Without considering the direct transmission, the outage probability [3] of user 1 is given by

$$
\begin{aligned}
P_{\text {out }, 1} & =\operatorname{Pr}\left\{\frac{1}{2} \min \left\{\log \left(1+\mathrm{SINR}_{S_{1} R_{l^{*}}}\right), \log \left(1+\mathrm{SNR}_{R_{l^{*}} D}\right)\right\}<\lambda\right\} \\
& =\operatorname{Pr}\left\{\gamma_{l^{*}, 1}<\gamma\right\},
\end{aligned}
$$

where $\lambda$ is the target rate and $\gamma=2^{2 \lambda}-1$. With best selection, we choose the relay $R_{l^{*}}$ such that $\gamma_{l^{*}, 1}=\max _{l} \gamma_{l, 1}$ and the outage probability can be written as

$$
P_{\text {out }, 1}=\operatorname{Pr}\left\{\max _{l} \gamma_{l, 1}<\gamma\right\}=\operatorname{Pr}\left\{\gamma_{l, 1}<\gamma, \forall l=1,2, \cdots, L\right\} .
$$

Since $\gamma_{l, 1}=\min \left(\frac{\left[\boldsymbol{\Gamma}_{l}\right]_{1,1}}{1-\left[\boldsymbol{\Gamma}_{l}\right]_{1,1}}, P\left|h_{R_{l} D}\right|^{2}\right)$, for $\boldsymbol{\Gamma}_{l}$ given in (16), we can see that the value of $\gamma_{l, 1}$ depends only on $h_{S_{1} R_{l}}, \cdots, h_{S_{K} R_{l}}$ and $h_{R_{l} D}$. Therefore, $\gamma_{1,1}, \gamma_{2,1}, \cdots, \gamma_{L, 1}$ are independent and the outage probability can be written as

$$
\begin{aligned}
P_{\text {out }, 1} & =\prod_{l=1}^{L} \operatorname{Pr}\left(\gamma_{l, 1}<\gamma\right)=\prod_{l=1}^{L}\left[1-\operatorname{Pr}\left(\gamma_{l, 1} \geq \gamma\right)\right] \\
& =\prod_{l=1}^{L}\left[1-\operatorname{Pr}\left\{\min \left(\frac{\left[\boldsymbol{\Gamma}_{l}\right]_{1,1}}{1-\left[\boldsymbol{\Gamma}_{l}\right]_{1,1}}, P\left|h_{R_{l} D}\right|^{2}\right) \geq \gamma\right\}\right] \\
& =\prod_{l=1}^{L}\left[1-\operatorname{Pr}\left(\frac{\left[\boldsymbol{\Gamma}_{l}\right]_{1,1}}{1-\left[\boldsymbol{\Gamma}_{l}\right]_{1,1}} \geq \gamma\right) \operatorname{Pr}\left(P\left|h_{R_{l} D}\right|^{2} \geq \gamma\right)\right](31)
\end{aligned}
$$

Notice that, from (16) and the fact that $\sigma_{n}^{2}=1$, we have $\boldsymbol{\Gamma}_{l}=$ $\mathbf{H}_{S R_{l}}^{H}\left(\mathbf{H}_{S R_{l}} \mathbf{H}_{S R_{l}}^{H}+\sigma_{n}^{2} \mathbf{R}^{-1}\right)^{-1} \mathbf{H}_{S R_{l}}=\left(\mathbf{I}+\mathbf{H}_{S R_{l}}^{-1} \mathbf{R}^{-1} \mathbf{H}_{S R_{l}}^{-H}\right)^{-1}$. Then, we can show that

$$
\begin{aligned}
\mathbf{I}-\boldsymbol{\Gamma}_{l} & \stackrel{(a)}{=} \mathbf{H}_{S R_{l}}^{-1}\left(\mathbf{R}+\mathbf{H}_{S R_{l}}^{-H} \mathbf{H}_{S R_{l}}^{-1}\right)^{-1} \mathbf{H}_{S R_{l}}^{-H} \\
& \stackrel{(b)}{=} \mathbf{H}_{S R_{l}}^{-1}\left[\rho \mathbf{e}_{K} \mathbf{e}_{K}^{T}+(1-\rho) \mathbf{I}_{K \times K}+\mathbf{H}_{S R_{l}}^{-H} \mathbf{H}_{S R_{l}}^{-1}\right]^{-1} \mathbf{H}_{S R_{l}}^{-H},
\end{aligned}
$$

where (a) follows from the matrix inversion lemma, which states that

$$
\left(\mathbf{A}+\mathbf{B} \mathbf{C B}^{H}\right)^{-1}=\mathbf{A}^{-1}-\mathbf{A}^{-1} \mathbf{B}\left(\mathbf{C}^{-1}+\mathbf{B}^{H} \mathbf{A}^{-1} \mathbf{B}\right)^{-1} \mathbf{B}^{H} \mathbf{A}^{-1},
$$

by taking $\mathbf{A}=\mathbf{I}, \mathbf{B}=\mathbf{H}_{S R_{l}}^{-1}, \mathbf{C}=\mathbf{R}^{-1}$ and (b) follows by rewriting the correlation matrix as $\mathbf{R}=\rho \mathbf{e}_{K} \mathbf{e}_{K}^{T}+(1-\rho) \mathbf{I}_{K \times K}$, where $\mathbf{e}_{K}$ is a $K \times 1$ all-1 vector. By applying the matrix inversion lemma again, with $\mathbf{A}=(1-\rho) \mathbf{I}_{K \times K}+\mathbf{H}_{S R_{l}}^{-H} \mathbf{H}_{S R_{l}}^{-1}$ (which is diagonal), $\mathbf{B}=\mathbf{e}_{K}$ and $\mathbf{C}=\rho$, we have

$$
\begin{aligned}
& \left(\mathbf{R}+\mathbf{H}_{S R_{l}}^{-H} \mathbf{H}_{S R_{l}}^{-1}\right)^{-1} \\
& =\operatorname{diag}\left(\kappa_{l, 1}, \cdots, \kappa_{l, K}\right)-\frac{\left[\kappa_{l, 1}, \cdots, \kappa_{l, K}\right]^{T}\left[\kappa_{l, 1}, \cdots, \kappa_{l, K}\right]}{\rho^{-1}+\sum_{k=1}^{K} \kappa_{l, k}}
\end{aligned}
$$

where $\kappa_{l, k}=\frac{P\left|h_{S_{k} R_{l}}\right|^{2}}{1+(1-\rho) P\left|h_{S_{k} R_{l}}\right|^{2}}$. Thus, it follows that the $(1,1)$ entry of $\mathbf{I}-\boldsymbol{\Gamma}_{l}$ is

$$
\left[\mathbf{I}-\boldsymbol{\Gamma}_{l}\right]_{1,1}=\frac{1}{P\left|h_{S_{1} R_{l}}\right|^{2}}\left(\kappa_{l, 1}-\frac{\kappa_{l, 1}^{2}}{\rho^{-1}+\sum_{k=1}^{K} \kappa_{l, k}}\right) .
$$

Moreover, since $\frac{\left[\boldsymbol{\Gamma}_{l}\right]_{1,1}}{\left[\mathbf{I}-\boldsymbol{\Gamma}_{l}\right]_{1,1}}=\frac{1}{\left[\mathbf{I}-\boldsymbol{\Gamma}_{l}\right]_{1,1}}-1$, we have

$$
\operatorname{Pr}\left(\frac{\left[\boldsymbol{\Gamma}_{l}\right]_{1,1}}{1-\left[\boldsymbol{\Gamma}_{l}\right]_{1,1}} \geq \gamma\right)=\operatorname{Pr}\left\{P\left|h_{S_{1} R_{l}}\right|^{2}\left(1-\rho+\frac{1}{\mathcal{V}_{M A I}}\right) \geq \gamma\right\},
$$

where

$$
\mathcal{V}_{M A I}=\frac{1}{\rho}+\sum_{k=2}^{K} \frac{P\left|h_{S_{k} R_{l}}\right|^{2}}{1+P\left|h_{S_{k} R_{l}}\right|^{2}(1-\rho)} .
$$

Note that $\mathcal{V}_{M A I}$ depends on the the channel from sources $S_{2}$, $S_{3}, \ldots, S_{K}$ to $R_{l}$. Assume that the correlation coefficient $\rho$ is strictly less than one. At high $\mathrm{SNR}$ (i.e., $P \gg 1$ ), $\mathcal{V}_{M A I}$ can be approximated as a deterministic variable $\mathcal{V}_{M A I} \approx \frac{1}{\rho}+\frac{K-1}{1-\rho}$ and the probability in (33) can be written as

$$
\operatorname{Pr}\left(\frac{\left[\boldsymbol{\Gamma}_{l}\right]_{1,1}}{1-\left[\boldsymbol{\Gamma}_{l}\right]_{1,1}} \geq \gamma\right) \approx \exp \left(-\frac{\gamma}{P} \frac{1+\rho(K-2)}{1+\rho(K-2)-\rho^{2}(K-1)}\right) \text {. }
$$

Finally, by substituting (34) into (31) and from the fact that $\operatorname{Pr}\left\{P\left|h_{R_{l} D}\right|^{2} \geq \gamma\right\}=\exp ^{-\gamma / P}$, since $h_{R_{l} D} \sim \mathcal{C N}(0,1)$, we have

$$
\begin{aligned}
P_{\text {out }, 1} & \approx \prod_{l=1}^{L}\left[1-\exp \left(-\frac{\gamma}{P} \frac{1+\rho(K-2)}{1+\rho(K-2)-\rho^{2}(K-1)}\right) \exp \left(-\frac{\gamma}{P}\right)\right] \\
& \approx\left[\frac{\gamma}{P} \frac{2(1-\rho)+\rho(2-\rho)(K-1)}{(1-\rho)[1+(K-1) \rho]}\right]^{L} \\
& =\exp \left\{-L \ln \left[\frac{P}{\gamma} \frac{(1-\rho)[1+(K-1) \rho]}{2(1-\rho)+\rho(2-\rho)(K-1)}\right]\right\} \\
& =\exp \left\{-L\left[\ln \left(\frac{P}{2^{2 R}-1}\right)+\ln C(\rho, K)\right]\right\},
\end{aligned}
$$

where $C(\rho, K)=\frac{(1-\rho)[1+(K-1) \rho]}{2(1-\rho)+\rho(2-\rho)(K-1)} \in[0,1)$ represents the performance degradation caused by the MAI. Hence, we have 
shown that the best selection strategy achieves diversity order equal to the number of relays $L$ even in the presence of MAI.

\section{REFERENCES}

[1] A. Sendonaris, E. Erkip, and B. Aazhang, "User Cooperation DiversityPart I: System Description" and "User Cooperation Diversity-Part II: Implementation Aspects and Performance Analysis," IEEE Trans. Commun., vol. 51, no. 11, Nov. 2003.

[2] J. Laneman and G. Wornell, "Distributed space-time-coded protocols for exploiting cooperative diversity in wireless networks," IEEE Trans. Inform. Theory, vol. 49, no. 10, pp. 2415-2425, Oct. 2003.

[3] J. Laneman, D. Tse, and G. Wornell, "Cooperative diversity in wireless networks: Efficient protocols and outage behavior," IEEE Trans. Inform. Theory, vol. 50, no. 12, pp. 3062-3080, Dec. 2004.

[4] A. Scaglione and Y.-W. Hong, "Opportunistic large arrays: Cooperative transmission in wireless multi-hop ad hoc networks for the reach back channel," IEEE Trans. Signal Processing, vol. 51, no. 8, Aug. 2003.

[5] A. Nosratinia, T. E. Hunter, and A. Hedayat, "Cooperative communication in wireless networks," IEEE Commun. Mag., vol. 42, no. 10, pp. 74-80, Oct. 2004.

[6] Y.-W. Hong, W.-J. Huang, F.-H. Chiu, and C.-C. J. Kuo, "Cooperative communications in resource-constrained wireless networks," IEEE Signal Processing Mag., vol. 24, no. 3, pp. 47-57, May 2007.

[7] M. Janani, A. Hedayat, T. E. Hunter, and A. Nosratinia, "Coded cooperation in wireless communications: Space-time transmission and iterative decoding," IEEE Trans. Signal Processing, vol. 52, no. 2, pp. 362-371, Feb. 2004.

[8] G. Kramer, M. Gastpar, and P. Gupta, "Cooperative strategies and capacity theorems for relay networks," IEEE Trans. Inform. Theory, vol. 51, no. 9, pp. 3037-3063, Sept. 2005.

[9] I. Hammerstroem, M. Kuhn, and A. Wittneben, "Impact of relay gain allocation on the performance of cooperative diversity networks," in Proc. IEEE Vehicular Technology Conference (VTC), 2004.

[10] M. Abdallah and H. C. Papadopoulos, "Beamforming algorithms for decode-and-forward relaying in wireless networks," in Proc. Conference on Information Sciences and Systems (CISS), 2005.

[11] Y. Jing and B. Hassibi, "Distributed space-time coding in wireless relay networks," IEEE Trans. Wireless Commun., vol. 5, no. 12, pp. 35243536, Dec. 2006.

[12] B. S. Mergen and A. Scaglione, "Randomized space-time coding for distributed cooperative communication," in IEEE International Conference on Communications (ICC), 2006.

[13] Y. Zhao, R. Adve, and T. Lim, "Improving amplify-and-forward relay networks: optimal power allocation versus selection," in Proc. IEEE International Symposium on Information Theory (ISIT), 2006.

[14] A. Bletsas, A. Khisti, D. P. Reed, and A.Lippman, "A simple cooperative diversity method based on network path selection," IEEE J. Select. Areas Commun., vol. 24, no. 3, pp. 659-672, Mar. 2006.

[15] S. Verdú, Multiuser Detection. Cambridge University Press, 1998

[16] R. Lupas and S. Verdú, "Linear multiuser detectors for synchronous code-division multiple-access channels," IEEE Trans. Inform. Theory, vol. 35, no. 1, pp. 123-136, Jan. 1989.

[17] U. Madhow and M. L. Honig, "MMSE interference suppression for direct-sequence spread-spectrum CDMA," IEEE Trans. Commun., vol. 42, no. 12, pp. 3178-3188, Dec. 1994.

[18] A. Duel-Hallen, "Decorrelating decision-feedback multiuser detection for synchronous code-division multiple-access channel," IEEE Trans. Commun., vol. 41, no. 2, pp. 285-290, Feb. 1993.

[19] J. G. Andrews, "Interference cancellation for cellular systems: A contemporary overview," IEEE Wireless Commun. Mag., vol. 12, no. 2, pp. 19-29, Apr. 2005.

[20] Y. Cao and B. Vojcic, "MMSE multiuser detection for cooperiave diversity CDMA systems," in Proc. Wireless Communications and Networking Conference (WCNC), 2004, pp. 42-47.

[21] L. Venturino, X. Wang, and M. Lops, "Multiuser detection for cooperative networks and performance analysis," IEEE Trans. Signal Processing, vol. 54, no. 9, pp. 3315-3329, Sept. 2006.

[22] B.R. Vojcic and W.M. Jang, "Transmitter Precoding in Synchronous Multiuser Communications," IEEE Trans. Commun., vol.46, no.10, pp. 1346-1355, Oct 1998

[23] Y. Jing and B. Hassibi, "Wireless networks, diversity and space-time codes," in Proceedings of IEEE Information Theory Workshop, Oct. 2004, pp. 463-468.
[24] J. Liu, J.-K. Zhang, and K. M. Wong, "On the design of minimum BER linear space-time block codes for MIMO systems equipped with MMSE receivers," IEEE Trans. Signal Processing, vol. 54, no. 8, pp. 3147-3158, Aug. 2006.

[25] S. Ulukus and R. Yates, "Optimum multiuser detection is tractable for synchronous CDMA systems using m-sequences," IEEE Commun. Lett., vol. 2, no. 4, pp. 89-91, Apr. 1998

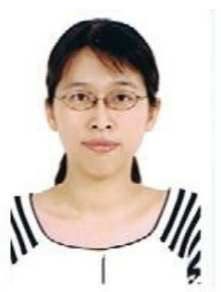

Wan-Jen Huang received her B.S. degree from the Department of Physics at National Taiwan University, Taipei, Taiwan, in 2001; and her M.S. degree from the Institute of Communication Engineering at National Taiwan University, Taipei, Taiwan, in 2003. She is currently working towards her Ph.D. degree in the Department of Electrical Engineering at the University of Southern California. Her research interests include precoding/equalizing for MIMO systems and signal processing techniques for cooperative networks.

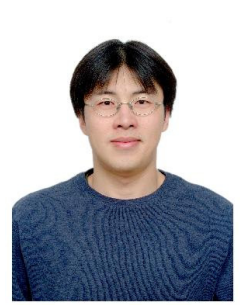

Y.-W. Peter Hong received his B.S. degree in Electrical Engineering from National Taiwan University, Taipei, Taiwan, in 1999, and his Ph.D. degree in Electrical Engineering from Cornell University, Ithaca, NY, in 2005. In Fall 2005, he joined the Institute of Communications Engineering/Department of Electrical Engineering at National Tsing Hua University, Hsinchu, Taiwan, where he is currently an Assistant Professor. His research interests include cooperative communications, distributed signal processing for sensor networks, and PHY-MAC crosslayer designs for next generation wireless networks. He received the best paper award among unclassified papers in MILCOM 2005 and the best paper award for young authors from the IEEE IT/COM Society Taipei/Tainan chapter in 2005 . He is a co-editor (along with A. Swami, Q. Zhao and L. Tong) of the book entitled "Wireless Sensor Networks: Signal Processing and Communications Perspectives" (John-Wiley, 2007).

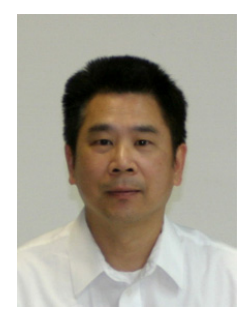

C.-C. Jay Kuo received the B.S. degree from National Taiwan University, Taipei, in 1980 and the M.S. and Ph.D. degrees from Massachusetts Institute of Technology, Cambridge, in 1985 and 1987, respectively, all in Electrical Engineering. Dr. Kuo was Computational and Applied Mathematics (CAM) Research Assistant Professor in the Department of Mathematics at the University of California, Los Angeles, from October 1987 to December 1988. Since January 1989 , he has been with the University of Southern California, where he is currently Professor of Electrical Engineering, Computer Science and Mathematics and Director of the Signal and Image Processing Institute. His research interests are in the areas of digital signal and image processing, multimedia compression, communication and networking technologies. Dr. Kuo has guided about 85 students to their Ph.D. degrees and supervised 20 postdoctoral research fellows. He is a co-author of about 140 journal papers, 730 conference papers and 9 books.

Dr. Kuo is a Fellow of IEEE and SPIE and a member of ACM. He is Editorin- Chief for the Journal of Visual Communication and Image Representation, and Editor for the Journal of Information Science and Engineering and the EURASIP Journal of Applied Signal Processing. He was on the Editorial Board of the IEEE Signal Processing Magazine in 2003-04. He served as Associate Editor for IEEE Transactions on Image Processing in 1995-98, IEEE Transactions on Circuits and Systems for Video Technology in 199597 and IEEE Transactions on Speech and Audio Processing in 2001-03. He received the National Science Foundation Young Investigator Award (NYI) and Presidential Faculty Fellow (PFF) Award in 1992 and 1993, respectively. 